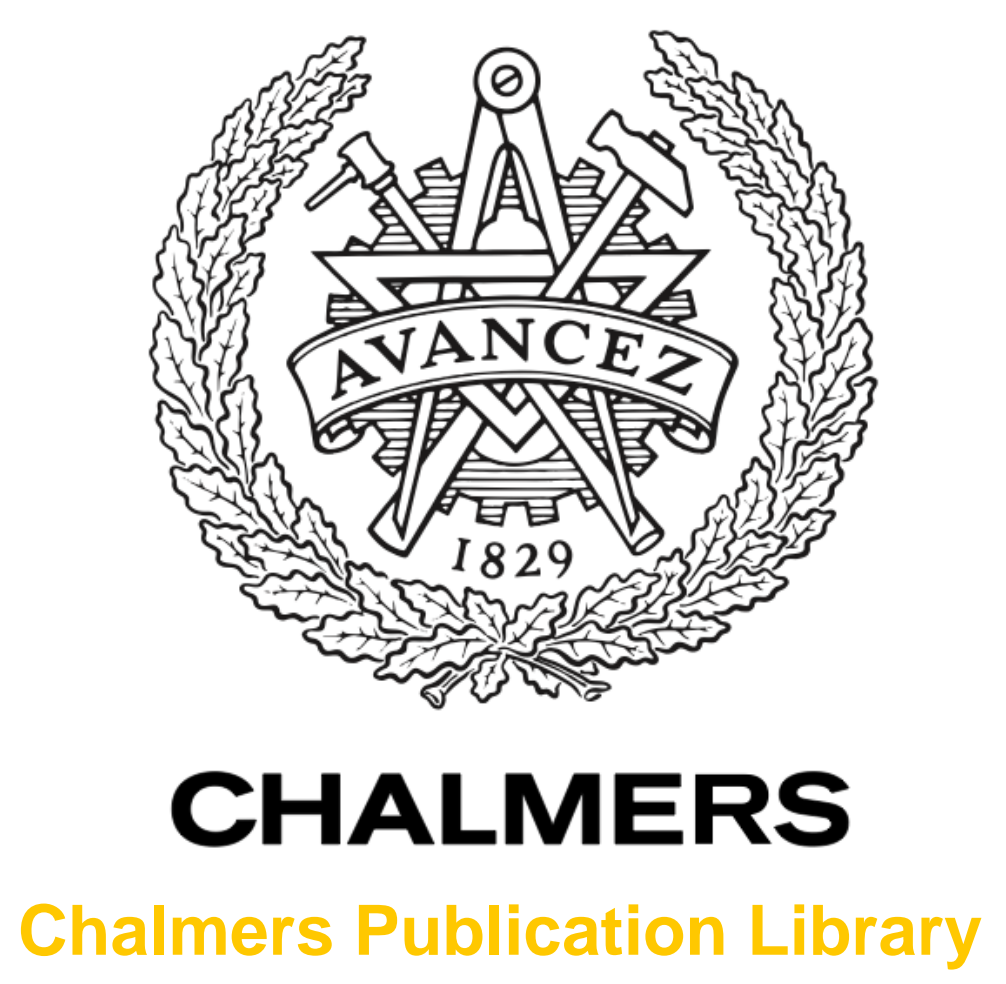

A Novel Demodulation Scheme for a Memoryless Optical Interference Channel

This document has been downloaded from Chalmers Publication Library (CPL). It is the author's version of a work that was accepted for publication in:

IEEE Int. Symp. Inf. Theory (ISIT), Aachen, Germany, June 2017.

Citation for the published paper:

Keykhosravi, K. ; Agrell, E. (2017) "A Novel Demodulation Scheme for a Memoryless

Optical Interference Channel". IEEE Int. Symp. Inf. Theory (ISIT), Aachen, Germany, June 2017.

Downloaded from: http://publications.lib.chalmers.se/publication/250959

Notice: Changes introduced as a result of publishing processes such as copy-editing and formatting may not be reflected in this document. For a definitive version of this work, please refer to the published source. Please note that access to the published version might require a subscription. 


\title{
A Novel Demodulation Scheme for a Memoryless Optical Interference Channel
}

\author{
Kamran Keykhosravi and Erik Agrell \\ Department of Signals and Systems, Chalmers University of Technology, 41296 Gothenburg, Sweden \\ Email: \{kamrank,agrell $\} @$ chalmers.se
}

\begin{abstract}
Matched filtering and sampling, which is known to be the optimal receiver for the linear additive white Gaussian noise channel, is in general suboptimal for a nonlinear medium. Nonetheless, it is commonly used in fiber-optical communication systems with nonlinear distortion. In this paper, a novel demodulation scheme is proposed for a two-user memoryless interference channel, with a type of nonlinear crosstalk that occurs in wavelength-multiplexed optical transmission. We show by simulations that by using this demodulation scheme, unlike matched filtering and sampling, the symbol error rate decreases to zero in the high-power regime.
\end{abstract}

\section{INTRODUCTION}

Coherent transmission over the fiber-optical channel is impacted by several impairments, primarily the Kerr nonlinearity. Due to the dependence of the optical fiber refractive index on signal intensity, the nonlinear Kerr effect causes a phase shift that depends on the amplitude of the transmitted signal itself. It plays a significant role at high transmit powers and critically limits the achievable data rates. The demodulation and detection mechanisms at an optical coherent receiver have been adopted from the wireless communication realm where channel nonlinearities are nonexistent, resulting in suboptimal solutions.

In today's optical networks, roughly one hundred wavelength-division-multiplex (WDM) signals copropagate in a standard single-mode fiber. The Kerr nonlinearity gives rise to crosstalk between the WDM channels. The signal phase is modulated in each WDM channel with its own amplitude as well as with the intensity of its neighboring channels. The former effect is referred to as self-phase modulation (SPM) and the latter as cross-phase modulation (XPM). Four-wave-mixing is another important nonlinear impairment that limits the performance of WDM systems [1, Sec. 8.5].

Due to the complicated interplay between the linear chromatic dispersion, the nonlinear Kerr effect, and the additive amplification noise, the capacity of the pointto-point optical fiber is still undisclosed. To compensate for the nonlinear interference, many solutions have been proposed, both in the electrical and in the optical realms [2, Ch. 4]. Digital back-propagation [3] at the receiver and signal predistortion at the transmitter [4] are wellknown impairment compensation methods that use digital signal processing. Optical solutions include soliton-based communication [5] and optical phase conjugation (see for example [6]). The performance of WDM systems can be improved by exploiting the temporal correlation of the nonlinear interference to mitigate the effects of XPM via an adaptive linear equalization (see for example [7]).

A large variety of simplified channel models have been considered in the literature (see [8]). Neglecting dispersion, modeling nonlinearity as a perturbation, and neglecting signal-noise interaction are among the central simplifying assumptions (see [9], [10], and [11] for examples). The study of simplified models can lead to an approximation of the channel behavior in certain power regimes as well as pave the way for further analysis on more realistic channels.

It is shown in [12] for a perturbative WDM model that the capacity region is hardly affected by the nonlinear inter-channel crosstalk if the information of all the channels is available at the receiver and the nonlinearity is weak. However, if joint detection is not possible, the capacity is extremely limited by the nonlinear crosstalk for the same model [12]. In [13] and [14] the capacity of a two-user simplified WDM system is studied as the input power goes to infinity. It is shown that the pre-log capacity 1 can be achieved for both channels.

Matched filtering and sampling are used in all of the aforementioned works to obtain a discrete-time model from a continuous channel. However, as mentioned before, this is suboptimal for the nonlinear optical channel. Here we study, by evaluating the symbol error rate (SER), the performance of a new demodulation scheme over a simplified two-user interference channel with nonlinear WDM crosstalk.

Contribution: In this paper, we study a pair of continuous-time WDM channels at practical power levels. First, we consider the conventional matched filtering and sampling method, which serves as a benchmark. Second, we modify this scheme by compensating for the SPM. Third, we propose a demodulation method tailored for the channel models under study, which mitigates the effects of both SPM and XPM. To compare the performance of the three demodulation algorithms, we evaluate the SER through Monte Carlo simulation. The simulation results show that while the performance of the first two methods degrades by increasing the input power beyond an optimal point, the SER for the third demodulation 
scheme decreases to zero in the high-power regime.

\section{Channel Model}

The signal propagation through an optical fiber can be modeled with the nonlinear Schrödinger (NLS) equation [15, Eq. 2.6.18] which accounts for the effects of chromatic dispersion and Kerr nonlinearity. Two types of amplification are used in the optical systems to compensate for the signal loss, namely, lumped and distributed amplification. While the latter amplifies the signal continuously through the fiber, the former does so only at the end of each fiber span. Here, we focus on lumped amplification. Amplifiers boost the signal power at the expense of adding noise.

If the fiber loss and polarization drift are neglected, the propagation of two single-polarization WDM fields with nonoverlapping spectra through a single-mode fiber can be described by a pair of coupled NLS equations [1, Eqs. 7.4.1 and 7.4.2]

$\frac{\partial A_{1}}{\partial z}+\frac{j \beta_{21}}{2} \frac{\partial^{2} A_{1}}{\partial t^{2}}-j \gamma_{1}\left(\left|A_{1}\right|^{2}+2\left|A_{2}\right|^{2}\right) A_{1}=0$
$\frac{\partial A_{2}}{\partial z}+d \frac{\partial A_{2}}{\partial t}+\frac{j \beta_{22}}{2} \frac{\partial^{2} A_{2}}{\partial t^{2}}-j \gamma_{2}\left(\left|A_{2}\right|^{2}+2\left|A_{1}\right|^{2}\right) A_{2}=0$ where $A_{k}=A_{k}(z, t)$ is the complex envelope of the optical field of channel $k \in\{1,2\}$ at time $t$ and position $z$. The constants $\gamma_{k}, \beta_{2 k}$, and $d$ denote the nonlinear, dispersion, and group velocity mismatch parameters, respectively.

In [13], a simplified two-user WDM system is studied based on (1)-(2), where the effects of dispersion, group velocity mismatch, and signal-noise interaction are neglected. The continuous-time network model can be described as [13, Eqs. (5) and (6)]

$$
\begin{aligned}
& A_{1}(L, t)=A_{1}(0, t) e^{j \eta_{1}\left(\left|A_{1}(0, t)\right|^{2}+2\left|A_{2}(0, t)\right|^{2}\right)}+N_{1}(t) \\
& A_{2}(L, t)=A_{2}(0, t) e^{j \eta_{2}\left(\left|A_{2}(0, t)\right|^{2}+2\left|A_{1}(0, t)\right|^{2}\right)}+N_{2}(t)
\end{aligned}
$$

Here, $L$ is the length of the fiber and $N_{k}(t)$ denotes the zero-mean white complex circularly symmetric Gaussian noise added by the inline amplifiers with spectral density $N_{0}=1 / 2 n_{\text {span }} h \nu F G$, where $h \nu$ is the optical photon energy, $n_{\text {span }}$ is the number of amplification spans, $T$ is the symbol time, and $F$ is the noise figure. Moreover, $G$ is the amplifier gain, which is equal to the signal attenuation in one span, $\exp \left(\alpha L_{\text {span }}\right)$, where $\alpha$ is the attenuation constant and $L_{\text {span }}=L / n_{\text {span }}$ is the span length. The parameter $\eta_{k}$ quantifies the nonlinear interference. In this paper we take into account the effect of fiber loss in the calculation of $\eta_{k}$ to obtain

$$
\eta_{k}=n_{\mathrm{span}} \gamma_{k} L_{\mathrm{eff}} .
$$

Here, $L_{\text {eff }}=\left(1-e^{-\alpha L_{\text {span }}}\right) / \alpha$ is the effective length of one fiber span [1, Eq. 4.1.6]. Due to the fiber loss, the signal power and consequently the nonlinear distortion diminish along propagation. Therefore, the effective length is less than the actual fiber length, $L_{\text {span }}$.

If we assume that perfect rectangular pulse shaping is used in the modulator, one can use matched filtering and sampling to obtain the following discrete-time network model from (3)-(4)

$$
\begin{aligned}
& Y_{1}=X_{1} \exp \left(j \eta_{1}\left(\left|X_{1}\right|^{2}+2\left|X_{2}\right|^{2}\right)\right)+N_{1} \\
& Y_{2}=X_{2} \exp \left(j \eta_{2}\left(\left|X_{2}\right|^{2}+2\left|X_{1}\right|^{2}\right)\right)+N_{2}
\end{aligned}
$$

where $N_{k}$ is zero-mean Gaussian noise with variance $P_{N}=$ $N_{0} / T$. Ghozlan and Kramer show in [13], through a highpower asymptotic analysis, that the capacity pre-log pair $(1,1)$ is achievable for the pair of channels $(6)-(7)$. The same authors extended this result in [14] to channels with group velocity mismatch. Here, we focus on the continuous channels (3)-(4) for a nonrectangular pulse shape in the practical power regime. We note that in $(3),(4),(6)$, and (7) the first term in the exponent is due to SPM, and the second term to XPM. It is assumed throughout the paper that independent data is transmitted over each channel and each receiver only has access to the output of its own channel. Both wavelengths are assumed to propagate through one point-to-point single mode fiber whose parameters are known at both receivers.

\section{Demodulation Schemes}

In this section, we list three demodulation schemes for the continuous-time network (3)-(4). Let the pulse shape $p(t)$ be a real function that is zero outside the interval $(0, T]$. Furthermore, define $A_{k}(0, t)=\sum_{i} x_{k, i} p(t-i T)$ to be the signal sent by the transmitter $k$, where $x_{k, i}$ indicates the $i$ th transmitted symbol.

The $k$ th receiver first demodulates the signal $A_{k}(L, t)$ (e.g. by passing it through a filter) and then detects the transmitted symbol. Throughout this paper, we assume that the detector selects the closest symbol in Euclidean distance to the demodulator output. Let $\tilde{y}_{k, i}$ be the output of the demodulator and $y_{k, i}$ be the detected symbol at the receiver. We present three demodulation schemes to obtain $\tilde{y}_{k, i}$ from the received signal $A_{k}(L, t)$.

1) Matched filtering and sampling (MFS): This scheme is the optimal demodulation method for the additive white Gaussian noise (AWGN) channel. The received signal is multiplied by the pulse shape and is integrated over time, i.e.,

$$
\tilde{y}_{k, i}^{\mathrm{mfs}}=\int_{-\infty}^{\infty} A_{k}(L, t) \cdot p(t-i T) \mathrm{d} t .
$$

2) Matched filtering and sampling with phase compensation $(M P C)$ : Here, we modify the MFS method by compensating for the phase rotation caused by SPM, i.e.,

$$
\tilde{y}_{k, i}^{\mathrm{mpc}}=\tilde{y}_{k, i}^{\mathrm{mfs}} e^{-j \eta_{k}\left|\tilde{y}_{k, i}^{\mathrm{mfs}}\right|^{2}},
$$

where $\tilde{y}_{k, i}^{\mathrm{mfs}}$ is the output of the MFS method. We note that $\mathrm{MPC}$ is common in optical receivers [16].

3) Maximum Matching ( $M x M)$ : Here, we propose a new demodulation technique tailored for (3)-(4). Our method is based on the following proposition, whose proof can be found in [17, Thm. 2.1]. 
TABLE I: Channel parameters used in the simulation.

\begin{tabular}{ccc}
\hline \hline Parameter & Symbol & Value \\
\hline Span length & $L_{\text {span }}$ & $85 \mathrm{~km}$ \\
Attenuation & $\alpha$ & $0.2 \mathrm{~dB} / \mathrm{km}$ \\
Nonlinearity & $\gamma_{1}=\gamma_{2}$ & $1.27(\mathrm{Wkm})^{-1}$ \\
Symbol rate & $1 / T$ & $32 \mathrm{Gbaud}$ \\
Optical photon energy & $h \nu$ & $1.28 \cdot 10^{-19} \mathrm{~J}$ \\
Amplifier noise figure & $F$ & $6 \mathrm{~dB}$ \\
Number of spans & $n_{\text {span }}$ & 5 \\
\hline \hline
\end{tabular}

Proposition 1: Let $f(t)$ be a positive continuous function on the interval $[a, b]$ and let $M(s)=\int_{a}^{b} f(t) e^{j s f(t)} \mathrm{d} t$, where $s$ is a real number. Then $\max _{s}|M(s)|=\int_{a}^{b} f(t) \mathrm{d} t$ and the maximum occurs if and only if $s \cdot f(t)$ does not depend on $t$, i.e., either $f(t)$ is a constant or $s$ is zero.

Inspired by this theorem, we propose the following demodulation method. Let

$s_{k, i}^{\max }=\underset{s \in B_{k}}{\arg \max _{-\infty}}\left|\int_{-\infty}^{\infty} A_{k}(L, t) \cdot p(t-i T) e^{-j \eta_{k} s(p(t-i T))^{2}} \mathrm{~d} t\right|$.

Here, the set $B_{k}$ comprises all possible values that $\left|x_{k, i}\right|^{2}+$ $2\left|x_{\bar{k}, i}\right|^{2}$ can take, where $\bar{k}=3-k$. The output of the maximum matching demodulator is defined as

$$
\tilde{y}_{k, i}^{\operatorname{mxm}}=\int_{-\infty}^{\infty} A_{k}(L, t) \cdot p(t-i T) e^{-j \eta_{k} s_{k, i}^{\max }(p(t-i T))^{2}} \mathrm{~d} t .
$$

Now, we justify the proposed demodulator with the help of Proposition 1. If noise is neglected, the right-hand side of (10) can be rewritten as

$$
\underset{s \in B_{k}}{\arg \max _{0, i}}\left|x_{-\infty}^{\infty} p^{2}(t) e^{j \eta_{k}\left(\left|x_{k, i}\right|^{2}+2\left|x_{\bar{k}, i}\right|^{2}-s\right) p^{2}(t)} \mathrm{d} t\right|
$$

By Proposition 1,s $=\left|x_{k, i}\right|^{2}+2\left|x_{\bar{k}, i}\right|^{2}$ achieves the maximum in (12). Conditional on $s_{k, i}^{\max }=\left|x_{k, i}\right|^{2}+2\left|x_{\bar{k}, i}\right|^{2}$, the interference gets canceled in the integrand in (11) and $\tilde{y}_{k, i}^{\mathrm{mxm}}$ follows a Gaussian distribution with mean $x_{k, i}$.

\section{Simulation Results}

In this section, we evaluate the three demodulation schemes listed in Section III by conducting Monte Carlo simulation. We consider the transmission of 16-ary quadrature amplitude modulation (QAM) data symbols for both transmitters. The dispersion is assumed to be zero to all orders. The channel parameters are presented in Table I. The nonlinear coefficient can be calculated using (5) as $\eta_{1}=\eta_{2}=135 \mathrm{~W}^{-1}$. The noise variance can be calculated as $P_{\mathrm{ASE}}=2 \mu \mathrm{W}$. We use 500 samples per symbol and a truncated Gaussian pulse shape with a full width at half maximum of $T / 2$. The input power, $P$, is assumed to be the same for both channels.
In Fig. 1, the distribution of the output of the three demodulators described in Sec. III for two input powers is depicted. With MFS and $P=0 \mathrm{dBm}$, in Fig. 1a, each constellation point is mapped to three noisy spots, each corresponding to one of the three possible values of the XPM term, $2\left|x_{2, i}\right|^{2}$. Moreover, because of SPM, these three spots are rotated with an angle proportional to the power of the constellation point.

At $10 \mathrm{dBm}$, with the MFS demodulation scheme as in Fig. 1d, the nonlinearity distorts not only the phase of the demodulator output, but also its amplitude. This observation can be explained as follows. Due to the nonlinearity, the received signal, $A_{k}(L, t)$, experiences a phase shift with respect to its amplitude at any time instance, $t$. Since $p(t)$ is not rectangular, in the high-power regime, the phase of $A_{k}(L, t)$ changes rapidly during one time slot. This rapid variation of the phase of the integrand in (8) scales down the output of the MFS demodulator.

At $0 \mathrm{dBm}$ with the MxM demodulator, it can be seen in Fig. 1c that although, like with MFS and MPC, each constellation point is mapped to three noisy spots, these spots are close to each other and centered at the transmitted point. As shown in Fig. 1f, in the high-power regime the effects of nonlinearity is completely compensated by the MxM demodulator and the output of the demodulator has almost the same distribution as the linear AWGN channel.

In Fig. $2 \mathrm{a}$, the SER $\operatorname{Pr}\left(y_{1, i} \neq x_{1, i}\right)$ for the three demodulation schemes presented in Sec. III are depicted. The SER for a linear additive white Gaussian noise (AWGN) channel with the noise variance $P_{\mathrm{ASE}}$ is included for comparison. The SER of the MFS demodulator is similar to that of the AWGN channel at low powers where the effects of the nonlinearity are weak and the channel can be assumed linear. However, at moderate power when the nonlinearity becomes substantial, the SER increases with power. Eventually, all the information is lost due to the nonlinear interference in the high-power regime.

Like MFS, the SER of MPC is close to the AWGN channel at low power, has a minimum in the moderate power regime, and increases to almost one at high power. However, since the SPM is compensated for in MPC, it outperforms MFS. The SERs at the optimal power for MPC and MFS are $1.6 \cdot 10^{-3}$ and $4 \cdot 10^{-3}$, respectively.

As the other two methods, the SER for MxM follows the AWGN channel when the input power is low. In the moderate-power regime at about $-7 \mathrm{dBm}$ the error probability increases as the effects of nonlinearity becomes substantial. However, unlike the MFS and MPC, the SER for MxM goes to zero at high power. When the input power grows large, the contribution of the noise to the righthand side of (10) becomes minimal and the approximation (12) becomes accurate. Therefore, the interference gets canceled by the MxM demodulator and $\tilde{y}_{k, i}^{\mathrm{mxm}}$ resembles the output of an AWGN channel (see Fig. 1f).

There is a peak in the SER approximately at $3 \mathrm{dBm}$ which can be interpreted as follows. There are two sources 


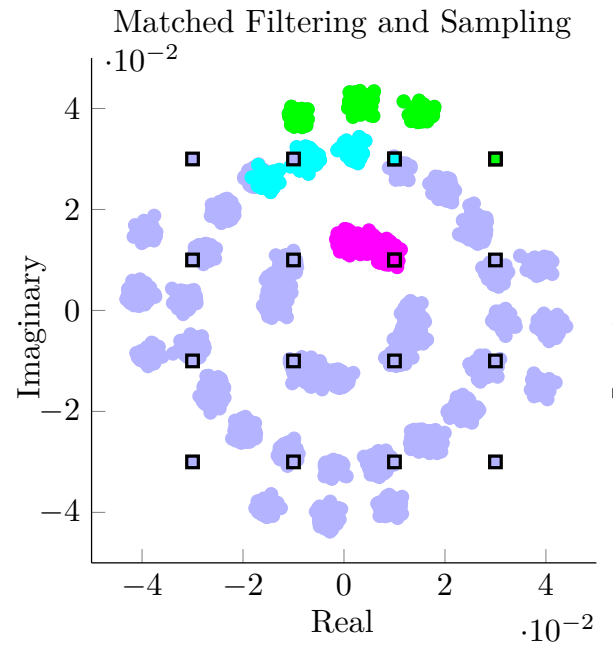

(a)

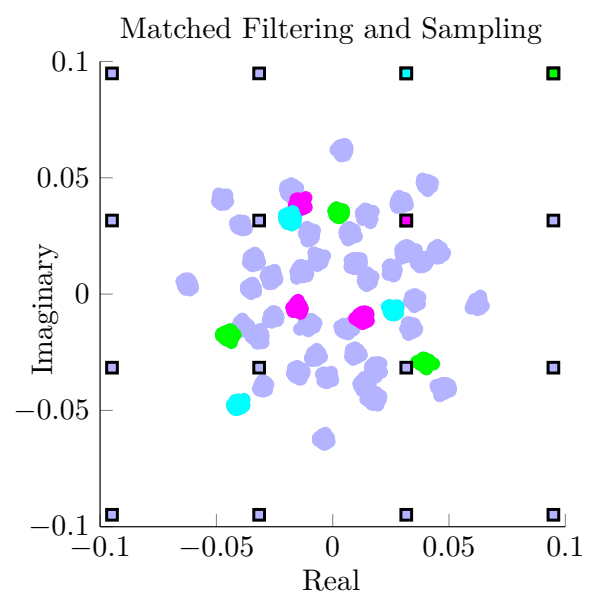

(d)

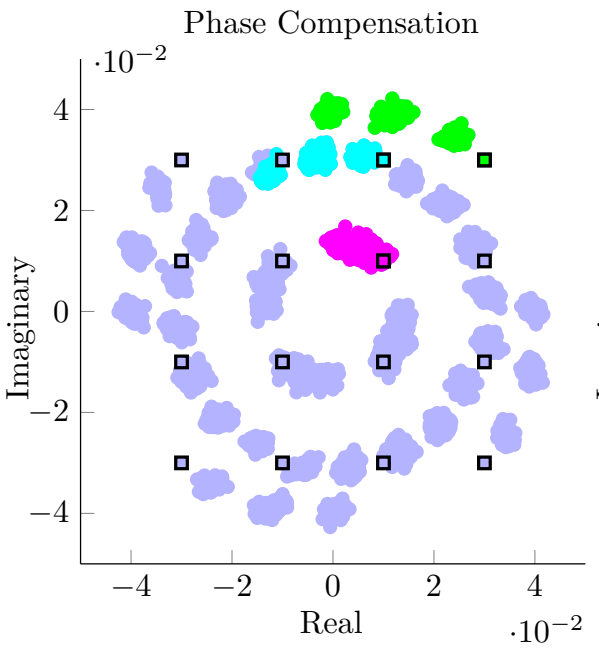

(b)

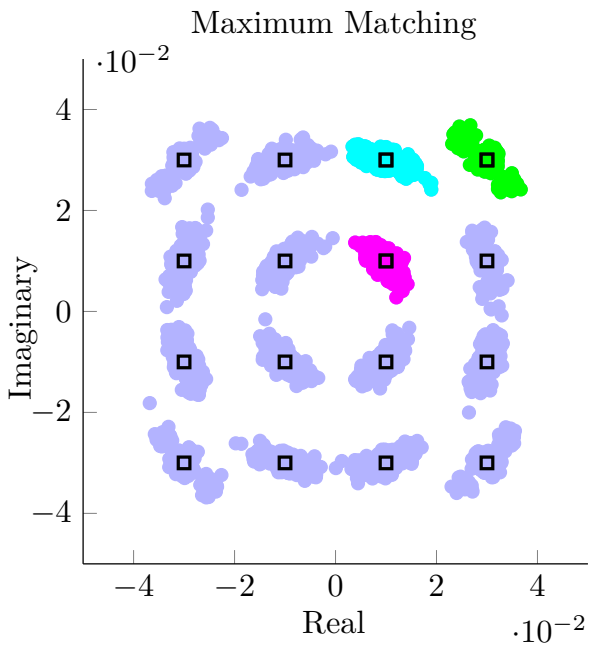

(c)

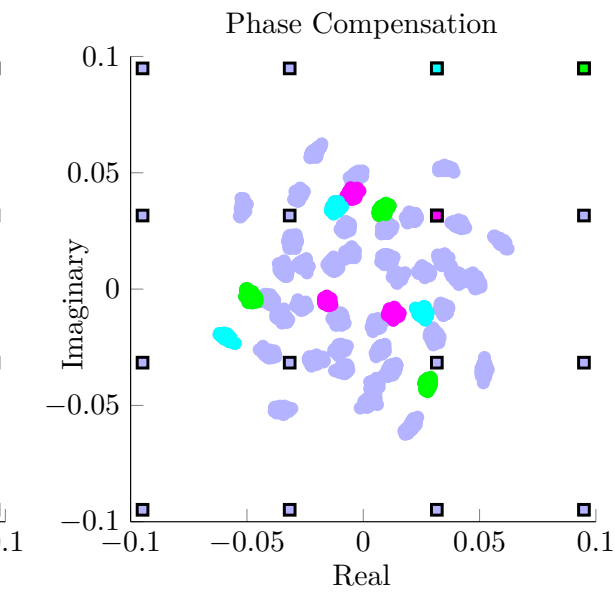

(e)

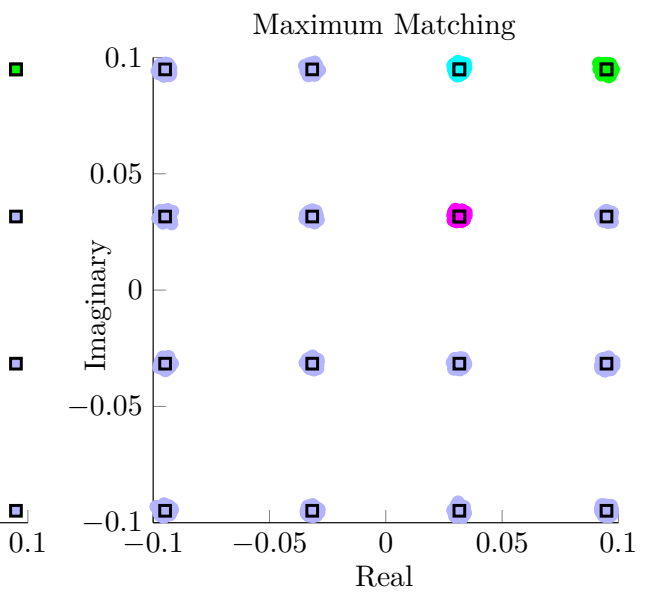

(f)

Fig. 1: The output of the demodulator (clouds) for 16-QAM (stars) using three demodulation schemes at signal power $P=0 \mathrm{dBm}(\mathrm{a})-(\mathrm{c})$ and $P=10 \mathrm{dBm}(\mathrm{d})-(\mathrm{f})$. Different colors are used to illustrate the scattering pattern of different constellation points.

of error when MxM is deployed: the error in finding $s_{k, i}^{\max }$ and the detection error. In (10), if because of the noise

$$
s_{k, i}^{\max } \neq\left|x_{k, i}\right|^{2}+2\left|x_{\bar{k}, i}\right|^{2},
$$

the outcome of the demodulator in (11) will not be centered at $x_{k, i}$ but at another point whose distance to $x_{k, i}$ increases with the input power. (The same behavior can be seen for MFS and MPC in Fig. 1.) Therefore the SER can suddenly increase at a power where the aforementioned center point exits the detection region of $x_{k, i}$. However, the SER goes to zero as the probability of the event described in (13) vanishes when the input power increases.

To further elucidate the proposed scheme's ability to suppress SPM and XPM by interference cancellation, in Fig. 2b, we evaluate $\operatorname{Pr}\left(s_{k, i}^{\max } \neq\left|x_{k, i}\right|^{2}+2\left|x_{\bar{k}, i}\right|^{2}\right)$. As can be seen, this probability decreases monotonically with the input power. Therefore, the success rate of the interference cancellation by the MxM demodulator converges to one at high power. We also note that by using MxM, receiver $k$, beside guessing $x_{k, i}$, can also obtain an approximation of $\left|x_{\bar{k}, i}\right|$ by calculating $\sqrt{\left(s_{k, i}^{\max }-\left|y_{k, i}^{\operatorname{mxm}}\right|^{2}\right) / 2}$. Therefore, one may exploit XPM to broadcast data over the network under study. This result suggests that treating XPM as noise in optical networks is suboptimal.

\section{Discussion and Conclusion}

A novel demodulation scheme, maximum matching, for a two-user optical interference channel was proposed. The matched filtering and sampling method with and without phase compensation were also considered for comparison. We showed via Monte Carlo simulation that for the maximum matching method, unlike the other two, the SER goes to zero as the power grows large. 


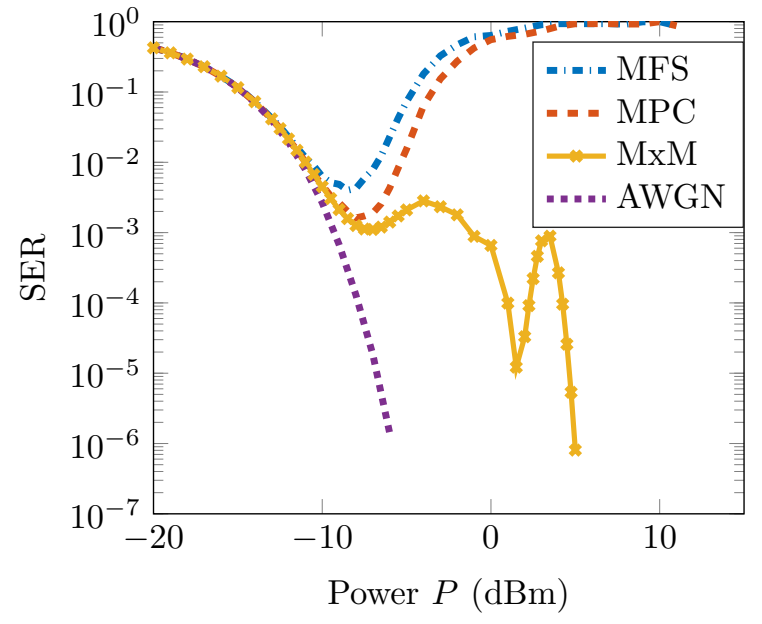

(a)

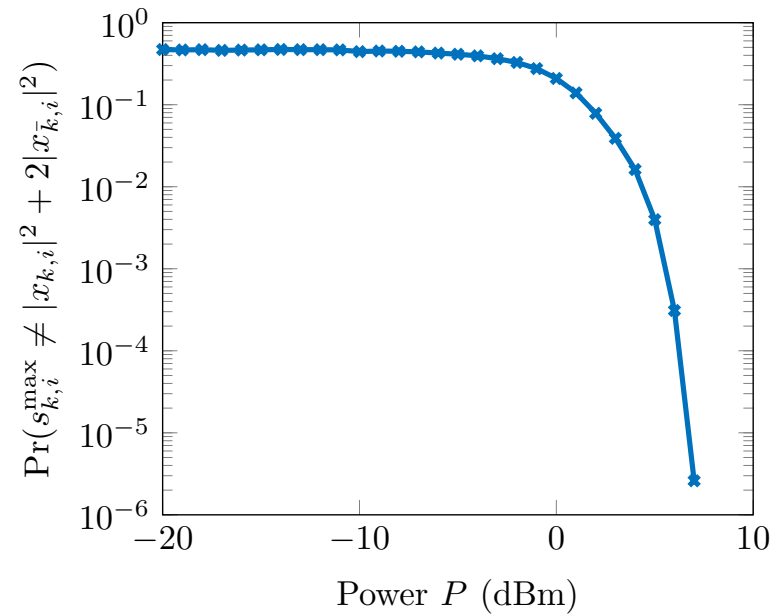

(b)

Fig. 2: (a) Symbol error rate $\operatorname{Pr}\left(y_{1, i} \neq x_{1, i}\right)$ evaluated by Monte Carlo simulation for 16-QAM data transmission over the twouser interference channel (3)-(4) for using matched filtering and sampling (MFS), matched filtering with phase compensation (MPC), and maximum matching (MxM). Also, the SER for the AWGN channel is depicted. (b): The error probability of the interference cancellation $\operatorname{Pr}\left(s_{k, i}^{\max } \neq\left|x_{k, i}\right|^{2}+2\left|x_{\bar{k}, i}\right|^{2}\right)$, using the MxM demodulation scheme.

A continuous-time communication channel can be described by a mapping whose range and domain have infinite dimensionality. By fixing a demodulation scheme we project the channel's output into a two dimensional complex number per each symbol interval. This mapping may result in information loss if the demodulation method has been selected poorly. It is well known that for the AWGN channel, the output of the matched filtering and sampling demodulator provides sufficient statistics [18, Thm. 26.4.1]. Our results indicate that deploying the matched filtering and sampling demodulator in nonlinear optical communication systems is strictly suboptimal. Future work should investigate the design of demodulation schemes for more realistic optical channel models.

\section{ACKNOWLEDGMENT}

The authors gratefully acknowledge helpful discussions with Giuseppe Durisi and support from the Swedish Research Council (VR) under grant no. 2013-5271.

\section{REFERENCES}

[1] G. P. Agrawal, Nonlinear Fiber Optics, 3rd ed. Academic Press, 2001.

[2] N. V. Irukulapati, "Towards the limits of nonlinearity compensation for fiber-optic channels," Ph.D. dissertation, Department of Signals and Systems, Chalmers University of Technology, Göteborg, 2016.

[3] E. Ip and J. M. Kahn, "Compensation of dispersion and nonlinear impairments using digital backpropagation," Journal of Lightwave Technology, vol. 26, no. 20, pp. 3416-3425, 2008.

[4] R. I. Killey, P. M. Watts, V. Mikhailov, M. Glick, and P. Bayvel, "Electronic dispersion compensation by signal predistortion using digital processing and a dual-drive mach-zehnder modulator," IEEE Photonics Technology Letters, vol. 17, no. 3, pp. 714$716,2005$.

[5] A. Hasegawa, "An historical review of application of optical solitons for high speed communications," Chaos: An Interdisciplinary Journal of Nonlinear Science, vol. 10, no. 3, pp. 475-485, 2000.
[6] S. Le, M. McCarthy, S. Turitsyn, I. Phillips, D. Lavery, T. Xu, P. Bayvel, and A. Ellis, "Optical and digital phase conjugation techniques for fiber nonlinearity compensation," in Proc. IEEE Opto-Electronics and Communications Conference (OECC), 2015.

[7] R. Dar, M. Feder, A. Mecozzi, and M. Shtaif, "Inter-channel nonlinear interference noise in WDM systems: modeling and mitigation," Journal of Lightwave Technology, vol. 33, no. 5, pp. 1044-1053, 2015.

[8] E. Agrell, G. Durisi, and P. Johannisson, "Information-theoryfriendly models for fiber-optic channels: A primer," in Proc. IEEE Inform. Theory Workshop, 2015.

[9] A. Mecozzi, "Limits to long-haul coherent transmission set by the Kerr nonlinearity and noise of the in-line amplifiers," $J$. Lightw. Technol., vol. 12, no. 11, pp. 1993-2000, 1994.

[10] B. Xu and M. Brandt-Pearce, "Modified Volterra series transfer function method," IEEE Photonics Technology Letters, vol. 14, no. 1, pp. 47-49, 2002.

[11] H. Song and M. Brandt-Pearce, "A discrete-time polynomial model of single channel long-haul fiber-optic communication systems," in Proc. IEEE Int. Conf. on Commun. (ICC), 2011.

[12] M. H. Taghavi, G. C. Papen, and P. H. Siegel, "On the multiuser capacity of WDM in a nonlinear optical fiber: Coherent communication," IEEE Trans. Inform. Theory, vol. 52, no. 11, pp. 5008-5022, Nov. 2006.

[13] H. Ghozlan and G. Kramer, "Interference focusing for mitigating cross-phase modulation in a simplified optical fiber model," in Proc. IEEE International Symposium on Inform. Theory, 2010, pp. 2033-2037.

[14] $\_$, "Interference focusing for simplified optical fiber models with dispersion," in Proc. IEEE International Symposium on Inform. Theory, 2011, pp. 376-379.

[15] G. P. Agrawal, Fiber-Optic Communication Systems, 3rd ed. John Wiley \& Sons, 2002.

[16] A. P. T. Lau and J. M. Kahn, "Signal design and detection in presence of nonlinear," J. Lightw. Technol., vol. 25, no. 10, pp. 3008-3016, Oct. 2007.

[17] C. Berg, Complex Analysis. University of Copenhagen, 2010.

[18] A. Lapidoth, A Foundation in Digital Communication. Cambridge University Press, 2009. 